'Escuela de Enfermería, Pontificia Universidad Católica de Chile, Santiago, Chile.

${ }^{2}$ Red de Salud UC ${ }^{3} U$ nidad de Infectología.

Complejo Asistencia Dr. Sótero del Río. aPhD, Enfermera-matrona. bEnfermera, Magíster en Ciencias de la Educación. 'Enfermera. ${ }^{\mathrm{d}}$ Matrona.

*Proyecto financiado por la Dirección de Investigación Escuela de Enfermería Pontificia Universidad Católica de Chile,

Concurso Interdisciplinario 2010 CIIEM\#201001 La organización que proporcionó el financiamiento no tuvo influencia en el diseño del estudio; en la recolección, análisis o interpretación de los datos; en la preparación, revisión o aprobación del manuscrito.

Recibido el 18 de Julio de 2012, aceptado el 17 de diciembre de 2012

Corespondencia a: Alejandra Araya Vicuña Mackenna 4860 Macul, Santiago, Chile. Teléfono: (56) 2 354-5834.

E-mail: aarayagu@uc.c

\section{Necesidades educativas de un grupo de personas viviendo con VIH: diagnóstico basado en la perspectiva de los usuarios y de los profesionales que los atienden}

\author{
ALEJANDRA ARAYA G. ${ }^{1, a}$, PAOLA CARRASCO A. ${ }^{1, b}$, \\ CARLA LOAYZA G. ${ }^{2, \mathrm{c}}$, ANA MARÍA FERNÁNDEZ S. ${ }^{3, \mathrm{~d}}$, \\ CARLOS PÉREZ C. ${ }^{2}$, MARTÍN LASSO B. ${ }^{3}$
}

\section{Identification of educational needs among patients with HIV and their health care providers}

Background: The success of educational interventions depends on the integration of educational programs into clinical practice. Aim: To determine the educational needs and perceived barriers of people living with HIV (PHIV) and their health care providers (HCP). Material and Methods: Qualitative study conducted in 60 PHIV and 10 HCP. For data collection, a semi-structured in-depth interview was applied, addressing the educational needs (content, methodology, person, time, physical location) and identified barriers to implement an educational program for PHIV. Content analysis technique was used for data analysis. Results: PHIV and their HCP identified the same educational needs as the following: general-related content, psychological, sexual and secondary prevention aspects of the disease. Individual sessions with written material and web pages were identified as important resources to support education. Both PHIV and professionals expressed their willingness to participate in educational programs, but the most commonly identified barrier was lack of time. Conclusions: This study identifies the key elements to include in an educational program for Chilean PHIV from the user and professional perspective.

(Rev Med Chile 2013; 141: 582-588).

Key words: Acquired immunodeficiency syndrome; Education.
E n Chile, estimaciones oficiales indican que en total existen 50.000 personas viviendo con el virus de la inmunodeficiencia humana (VIH) y con el síndrome de inmunodeficiencia adquirida (SIDA) ${ }^{1}$. El grupo de edad más afectado se encuentra entre los 20 y 49 años de edad, siendo el género masculino el más prevalente ${ }^{2}$. La mayoría de las personas que viven con VIH/SIDA (PVVIH) viven el Santiago y son atendidos por el sistema público de salud ${ }^{3}$.

En el año 2004, el acceso al tratamiento antirretroviral (TARV) alcanzó 100\% y en al año 2005 el VIH fue incorporado a las Garantías Explícitas en Salud (GES), garantizando su diagnóstico oportuno, TARV, exámenes de laboratorio y genotipificación según la condición del usuario ${ }^{4}$. El impacto de la TARV se ha visto reflejado en indicadores de salud tales como: disminución de la mortalidad, hospitalizaciones, retraso de la presentación del SIDA, de infecciones oportunistas y de las complicaciones derivadas de la enfermedad ${ }^{5-7}$. Estos indicadores son comparables con países desarrollados en los cuales el VIH es considerado una enfermedad crónica.

Como toda enfermedad crónica, en la cual la cura aún es desconocida, es indispensable apoyar a los usuarios con programas educativos destinados a disminuir la carga de la enfermedad, potenciar 
Necesidades educativas en personas viviendo con VIH - A. Araya et al

conductas de salud saludables, y disminuir los costos en salud asociados a la enfermedad ${ }^{8-10}$. El punto de partida de cualquier programa educativo es la evaluación y priorización de las necesidades educativas en términos del contenido, metodología, lugar, momento y persona más idónea para realizar un programa educativo destinado a PVVIH pueden contribuir a diseñar programas educativos más efectivos ${ }^{11,12}$. El objetivo de este estudio es conocer las necesidades educativas (contenidos, metodología, persona, momento, lugar físico) y las barreras percibidas de las PVVIH desde la perspectiva de los usuarios y de los profesionales de la salud que los atienden en dos centros de atención de PVVIH, con el propósito de planificar programas educativos basados en las necesidades de los usuarios.

\section{Material y Método}

Estudio descriptivo con entrevistas en profundidad, realizado en un total de 65 PVVIH y 10 profesionales del área de salud pertenecientes a unidades de infectología de la Región Metropolitana. El estudio fue realizado en el Complejo Asistencial Dr. Sótero del Río (CASR) perteneciente al sistema público de atención y en el Centro Médico San Joaquín (CMSJ) de la Red de Salud UC.

Los criterios de inclusión para las PVVIH fueron: a) ser mayor de 18 años; b) con diagnóstico confirmado de VIH; c) sin discapacidad mental; d) no haber estado hospitalizado el mes anterior, ya que las necesidades educativas pueden estar afectadas; y e) si es mujer, no estar embarazada, dado a que la condición de gestación puede influir en las necesidades educativas. Los criterios de inclusión para los profesionales de la salud fueron: a) tener contacto directo con usuarios con PVVIH, b) tener al menos $22 \mathrm{~h}$ de contrato en alguna de las instituciones involucradas en este estudio, c) tener una antigüedad de un año trabajando en contacto directo con PVVIH. Esta investigación contó con la aprobación de los Comités de Ética de la Escuela de Enfermería de la Pontificia Universidad Católica de Chile y del CASR.

La recolección de los datos fue realizada entre los meses de julio y diciembre de 2011. Se invitaron a participar a todos las PVVIH que acudieron a control médico en ese período de tiempo y que cumplieron con los criterios de inclusión. Posterior a la firma del consentimiento, se inició la entrevista en profundidad, en una sala privada destinada especialmente para este propósito, las que duraron en promedio 45 min y fueron grabadas completamente, para posteriormente ser transcritas de manera textual. Para el desarrollo de la entrevista en profundidad se usó una guía semiestructurada con cinco preguntas relacionadas a las necesidades educativas y dos preguntas sobre las barreras para implementarla (Tabla 1).

Para el análisis de los datos se utilizó la técnica de análisis de contenido de Krippendorff ${ }^{13}$. Este

Tabla 1. Preguntas sobre las necesidades educativas y las barreras percibidas por las PVVIH y los profesionales que los atienden para implementar un programa educativo en la práctica clínica

\begin{tabular}{|c|c|}
\hline \multicolumn{2}{|c|}{ Necesidades educativas } \\
\hline Contenidos & $\begin{array}{l}\text { ¿Cuáles son los contenidos elementales que debieran entregarse en un programa educativo destinado } \\
\text { a personas viviendo con VIH/SIDA? }\end{array}$ \\
\hline Metodología & $\begin{array}{l}\text { Con respecto a la metodología, ¿cómo cree usted que sería la mejor forma de entregar los contenidos } \\
\text { educativos a las personas viviendo con VIH/SIDA? }\end{array}$ \\
\hline Persona & $\begin{array}{l}\text { En su opinión, ¿quién o quiénes son las personas más idóneas para entregar los contenidos educa- } \\
\text { tivos? }\end{array}$ \\
\hline Momento & $\begin{array}{l}\text { A su juicio, ¿en qué momento de la enfermedad es necesario entregar y/o apoyar a las personas } \\
\text { viviendo con VIH/SIDA con contenidos educativos? }\end{array}$ \\
\hline Lugar & En su opinión, ¿cuál sería el lugar físico más idóneo para entregar estos contenidos educativos? \\
\hline \multicolumn{2}{|c|}{ Las barreras percibidas para participar de un programa educativo } \\
\hline Barreras del usuario & $\begin{array}{l}\text { Según su experiencia, ¿cuál o cuáles serían las barreras de las personas viviendo con VIHISIDA pueden } \\
\text { tener para participar de un programa educativo? }\end{array}$ \\
\hline $\begin{array}{l}\text { Barreras del } \\
\text { profesional }\end{array}$ & $\begin{array}{l}\text { Según su experiencia, ¿cuál o cuáles serian las barreras que usted como profesional de la salud que } \\
\text { atiene a PVVIH tendría para incorporar un programa educativo en su práctica clínica? }\end{array}$ \\
\hline
\end{tabular}


proceso consistió en la lectura de las entrevistas por separado, a cargo de tres investigadores. Primero se hizo una lectura de las entrevista sin interpretarlas; luego se realizó una segunda lectura con el objetivo de identificar las temáticas; y finalmente, se realizo una tercera lectura de cada una de las entrevistas para corroborar las temáticas identificadas por cada uno de los investigadores por separado. Luego cada investigador propuso su análisis y se llegó a un consenso de las temáticas encontradas.

Los resultados obtenidos fueron devueltos a 12 usuarios de los centros de atención en donde se desarrolló el estudio para corroborar la veracidad de estos y acoger sus opiniones al respecto. Además se realizó una reunión con expertos para la cual fueron convocados 12 profesionales médi$\cos$, matronas, enfermeras-matronas, enfermeras, asistente social, psicólogas con experiencia tanto clínica como temática en el área de la educación en PVVIH, con la finalidad de asegurar la credibilidad de los resultados encontrados y recoger sus sugerencias sobre la forma más adecuada de implementar un programa educativo en la práctica clínica de las unidades de infectología del sector público y privado de nuestro país.

\section{Resultados}

Para PVVIH, la mediana de edad fue 38 , con un mínimo de 21 y máximo de 77 años. 56/65 eran hombres y el resto mujeres, 36/65 eran hombres que tienen sexo con hombres, 20/65 heterosexuales y el resto bisexuales. 36/65 reportaban tener estudios universitarios/técnicos, 18/65 enseñanza media completa y el resto enseñanza básica. 49/65 reportaban tener algún tipo de trabajo remunerado, 10/65 eran cesantes, 4/65 jubilados y el resto no respondió. En relación a su enfermedad, 36/65 fueron diagnosticados en etapa de SIDA y el resto en etapa de $\mathrm{VIH}$; 55/65 se encontraban en tratamiento TARV, con una mediana de 36 meses de tratamiento.

En relación a los profesionales, la mediana de edad fue de 38 años, con un mínimo de 31 y máximo de 53 años. 6/10 eran mujeres y el resto hombres. 8/10 referían tener estudios de postgrado y trabajaban jornada completa, mientras el resto tenía formación universitaria de pregrado y trabajaba tiempo parcial en sus respectivos centros de salud.

Las opiniones de las entrevistas en profundidad se examinaron en sus aspectos esenciales y se presentan separadamente para cada aspecto educativo consultado (Tabla 2). Dado que no existieron diferencias entre las opiniones de las PVVIH y de los profesionales de la salud que los atendían, los resultados se presentan en conjunto para ambos participantes.

Tabla 2. Temáticas identificadas sobre las necesidades educativas desde la perspectiva de las PVVIH y de los profesionales que los atienden $(n=70)$

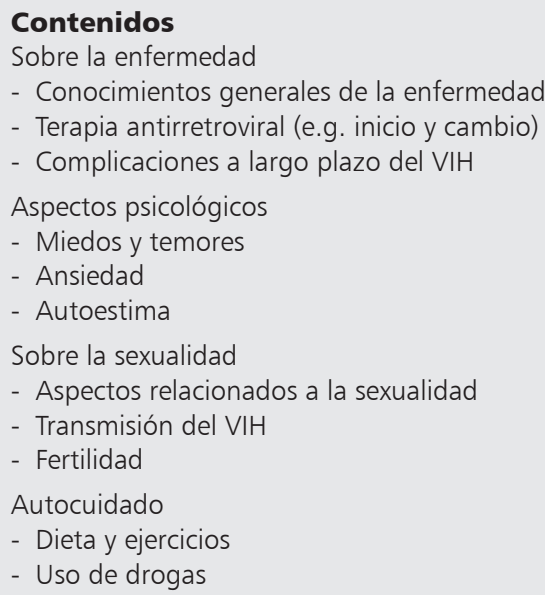

\section{Metodología}

Individual y planificada

Charlas grupal (6 ó 8 personas)

Medios audiovisuales

- Escrito (folleto, cuadernos, etc.)

- Uso de la tecnología (páginas web)

\section{Persona más idónea}

Profesionales del área de la salud

Médico

Matrona

Enfermera

Psicólogo

Otros: Personas viviendo con VIH

\section{Momento de la enfermedad}

Al principio

Desde el diagnóstico (consejería posttest)

Durante toda la enfermedad

Programada según los hitos de la enfermedad (e.g. inicio TARV)

Ocasiones especiales

Según necesidad del usuario

\section{Lugar}

En el mismo centro médico

Sala acogedora 
Los contenidos educativos se refieren a todos aquellos relatos en los cuales los usuarios (as) y los profesionales que los atienden han identificado las materias importantes de abordar que un programa educativo destinado a PVVIH. Ellos reconocen cuatro aspectos educativos distintivos: relativos a la enfermedad, aspectos psicológicos, sexualidad y autocuidado. En relación a la enfermedad, destacan relatos relacionados a incorporar contenidos de los aspectos generales de la enfermedad como son el significado de los exámenes de laboratorio que se controlan a lo largo de la enfermedad, como son CD4 y la carga viral, así como también, contenidos educativos relacionados a las complicaciones a largo plazo del VIH en relación a la aparición de infecciones oportunistas y de enfermedades no relacionadas al VIH. A su vez, cuando las PVVIH han iniciado TARV necesitan saber en qué consiste la acción de las drogas, así como también sus efectos secundarios a corto y mediano plazo.

Con relación a los aspectos psicológicos, se destacan aquellos contenidos relacionados a los miedos y temores, el manejo de la ansiedad que produce el vivir con esta enfermedad y al apoyo para fortalecer su autoestima. En relación a los contenidos de sexualidad, se destaca importantemente todos aquellos aspectos relacionadas a evitar la transmisión de la enfermedad a personas sero-desconocidas a través de conductas sexuales saludables. Cabe destacar que la temática de una futura fertilidad aparece en mujeres y hombres que teniendo su enfermedad bajo control, se cuestionan la posibilidad de ser padres biológicos. Finalmente, entre los aspectos relacionados con el autocuidado se encuentran el tipo de dieta y ejercicios más apropiados, uso de drogas lícitas como el alcohol e ilícitas como la marihuana.

Tanto los usuarios como los profesionales de la salud identifican que un programa educativo debiera tener una metodología individual y grupal. La educación individual planificada es identificada como una forma de entregar contenidos más sensibles como, por ejemplo, conductas sexuales de riesgo, en los cuales las PVVIH necesitan un espacio de confianza para discutir su experiencia; no así la forma grupal, la cual la ven como un complemento de la forma individual, en la cual identifican contenidos de dominio público, tales como los aspectos generales de la enfermedad. Cabe destacar que la posibilidad de intercambiar experiencia con otras PVVIH se transforma en una de las principales fortalezas de la metodología grupal. Dentro de los medios audiovisuales se destacan material escrito como folletos y el desarrollo de páginas web en la cual se encuentre la misma información que reciba de manera individual o grupal como forma de respaldo de los contenidos.

Los profesionales de la salud que los atienden son identificados como las personas más idóneas para entregar la educación, entre los cuales se destacan: el médico especialista en infectología, la matrona, la enfermera y la psicóloga. A su vez, las PVVIH identifican a otros pares, es decir, PVVIH que pueden incorporarse para reforzar los contenidos educativos entregados por los profesionales desde la perspectiva de la experiencia de otra PVVIH.

En relación al momento durante la enfermedad más adecuado para otorgar una educación, son claros en identificar que el momento del diagnóstico de la enfermedad es uno de los momentos más importantes de apoyar con contenidos educativos claros y precisos. Es uno de los períodos más frágiles, en los cuales los usuarios se ven enfrentados a sus miedos, que los hacen muchas veces alejarse de los controles de salud. Otros momentos identificados se relacionan a hitos durante toda la enfermedad como, por ejemplo, cuando se inicia la TARV. La segunda de ellas, se relaciona directamente con las necesidades emergentes de los usuarios, por ejemplo, en aquellos que quieren ser padres/madres.

El lugar físico más idóneo para entregar los contenidos educativos, es el centro médico donde reciben su atención de salud. En este sentido, ellos identifican una sala acogedora como el requerimiento necesario para poder desarrollar un programa educativo.

Con respecto a las barreras para implementar un programa educativo en el centro de salud, tanto los profesionales como las PVVIH identifican la falta de tiempo como la gran barrera (Tabla 3). Por su lado los profesionales refieren no tener tiempo para desarrollar un programa educativo en el tiempo que tienen asignado para ver a los usuarios y, los usuarios, por su parte identifican que el acudir al centro en un momento distinto al de su atención de salud puede dificultar su adherencia a un programa educativo. A su vez, las PVVIH identifican la estigmatización del grupo de educación, por parte de personas ajenas al programa de infectología, como una barrera para acceder a un programa educativo. 
Tabla 3. Dimensiones y significados identificados sobre las barreras percibidas para participar de un programa educativo

Barreras de las PVVIH de ambos centros

$$
(n=60)
$$

Barreras de los profesionales de la salud de ambos centros $(n=10)$
Falta de tiempo

Estigmatización de las PVVIH

Falta de tiempo

\section{Discusión}

A pesar de que existen publicaciones con estudios de evaluación de las necesidades educativas de PVVIH, ninguno de éstos ha incorporado usuarios Latinoamericanos y, en lo particular, esta área temática no ha sido objeto de publicación en Chile.

A nivel mundial el foco de los programas educativos históricamente ha estado centrado en educar personas que se presumen son VIH negativas, específicamente, en aumentar la comprensión de cómo ayudar a las personas a protegerse de contraer la infección por $\mathrm{VIH}^{14,15}$ y focalizados en la prevención primaria del VIH en poblaciones de alto riesgo, tales como usuarios de drogas endovenosas, hombres que tienen sexo con hombres, trabajadoras sexuales y en la prevención de la transmisión vertical ${ }^{16}$.

Sólo en los últimos años ha habido un desarrollo cada vez mayor de programas educativos orientados a PVVIH ${ }^{17}$. Estos programas educativos han estado centrados en disminuir conductas de riesgo sexual y de transmisión venosa ${ }^{18-20}$, así como también en mejorar la salud mental e inmunológica de las personas infectadas con $\mathrm{VIH}^{21-27}$. Las intervenciones educativas destinadas a PVVIH han tenido efectos contradictorios ${ }^{28-31}$, sugiriendo que el éxito de los programas educativos está centrado en una visión más comprensiva del autocuidado como, por ejemplo, en la evaluación de las necesidades educativas y de los obstáculos que enfrentan los usuarios para adherir a los programas educativos ${ }^{32}$.

Los resultados de este estudio identifican que las PVVIH tienen un gran interés de recibir educación desde que reciben el diagnóstico de VIH, lo que concuerda con lo reportando por otros investigadores ${ }^{12,19,33}$. Los usuarios manifiestan interés en aprender temas tales como alimentación saludable, manejo del estrés, conocimientos sobre la enfermedad y exámenes de laboratorio (carga viral y las células T), comunicación con otras personas sobre el VIH, cómo hacer frente a la incertidumbre de vivir con VIH, manejo de síntomas asociados a la enfermedad ${ }^{12,33}$. Las PVVIH confían en recibir educación de los profesionales de la salud que los atienden $^{34,35}$ en los centros de salud en los cuales reciben tratamiento.

Con respecto a las barreras identificadas, el tiempo es la barrera más destacada desde la perspectiva de las PVVIH y de los profesionales. Este es un hallazgo que no concuerda con otras investigaciones, las que identifican la dificultad de acceso a la información como la barrera más común para recibir educación ${ }^{36}$. La segunda barrera identificada por las PVVIH es la estigmatización de las personas que asisten a un programa educativo. Este punto es particularmente cierto para PVVIH en los cuales el estigma asociado a la enfermedad puede ser un obstáculo para participar de programas educativos ${ }^{10,11,33}$.

$\mathrm{Si}$ bien este estudio tiene las condiciones propias que no permite generalizar sus conclusiones, es el primer estudio chileno que aborda las necesidades educativas desde la perspectiva de las PVVIH y de los profesionales de la salud que los atienden que muestra la realidad de un centro público y privado de atención.

Beneficios para la salud pública de contar con programas educativos centrado en las necesidades educativas de las PVVIH es la potencialidad de mejorar indicadores de salud, disminución de costos asistenciales, así como también la disminución de personas contagiadas con VIH a través de personas que conocen su serología positiva ${ }^{8,27}$. Integrar entonces un programa educativo se vuelve un desafío y también una necesidad.

El éxito de las intervenciones educativas en las personas que están viviendo con VIH, dependerá de la integración de los programas educativos en la práctica clínica ${ }^{37-39}$. A pesar de que en la Guía Clínica de VIH/SIDA 2010 se establece la importancia de la atención integral, actualmente el Programa Garantías Explicitas en Salud de VIH/ SIDA no garantiza la educación a los usuarios 
como una prestación básica de la canasta. Así como sucede en para otras enfermedades crónicas, es necesario incorporar la prestación de educación a los usuarios a la canasta. De esta forma se inyectarían recursos económicos que ayudarían a aliviar la falta de tiempo que actualmente los profesionales identifican como la barrera para la implementación de programas educativos dentro de su práctica clínica.

\section{Referencias}

1. MINSAL. Situación epidemiológica de VIH/SIDA, Chile 1984-2008.2009: Disponible en: http://epi.minsal.cl/epi/ html/bolets/reportes/VIH-SIDA/InformePais_84-08_ vih_sida.pdf.

2. Pérez C, Vidal P, Dorman KS, Wang G, Wang G, Abarca $\mathrm{K}$, et al. Epidemiología molecular del virus de inmunodeficiencia humana tipo 1 en Santiago, Chile. Rev Med Chile 1999; 127: 1497-500.

3. Martínez P, Olea A, Chiu M. Situación epidemiológica de la infección por VIH y el síndrome de inmunodeficiencia adquirida en Chile. Rev. Chil. Infectol 2006; 23 (4): 321-9.

4. MINSAL. Guía clínica: Síndrome de inmunodeficiencia adquirida VIH/SIDA. Rev. Chil. Infectol 2010; 27 (3): 239-76.

5. Wolff C, Alvarado R, Wolff M. Prevalencia, factores de riesgo y manejo de la depresión en usuarios con infección por VIH: Revisión de la literatura. Rev. Chil. Infectol 2010; 27 (1): 65-74.

6. Wolff MJ, Beltrán CJ, Vásquez P, Ayala MX, Valenzuela M, Berríos G, et al. The Chilean AIDS cohort: A model for evaluating the impact of an expanded access program to antiretroviral therapy in a middle-income country-organization and preliminary results. J Acquir Immune Defic Syndr 2005; 15; 40 (5): 551-7.

7. Wolff MJ, Diomedi A, Morales O, Bidar T, Dabanch J, Bustamante C, et al. Seguimiento prospectivo de una población infectada por VIH con y sin posibilidades de terapia anti-retroviral: Impacto en sobrevida y complicaciones. Rev Med Chile 2001; 129: 886-94.

8. Lorig KR. Taking patient to the next level. RN 2003; 66 (12): 35-8.

9. Lorig KR, Sobel DS, Ritter PL, Laurent D, Hobbs M. Effect of a self-management program on patients with chronic disease. Eff Clin Pract 2001; 4 (6): 256-62.

10. Lau JT, Tsui HY, Li CK, Chung RW, Chan MW, Molassiotis A. Needs assessment and social environment of people living with HIV/AIDS in Hong Kong. AIDS Care 2003; 15 (5): 699-706.
11. Molassiotis A, Callaghan P, Twinn SF, Lam SW. Assessment of needs of adult symptomatic HIV patients in Hong Kong. AIDS Care 2001 Apr; 13 (2): 177-89.

12. Huber JT, Cruz JM. Information needs and informationseeking behaviors of HIV positive men and women. Med Ref Serv Q. 2000; 19 (3): 39-48.

13. Krippendorff K. Content Analysis: An Introduction to Its Methodology. Thousand Oaks, California: SAGE Publications; 2003.

14. Kelly JA, Kalichman SC. Behavioral research in HIV/ AIDS primary and secondary prevention: recent advances and future directions. J Consult ClinPsychol 2002; 70 (3): 626-39.

15. Janssen RS, Holtgrave DR, Valdiserri RO, Shepherd M, Gayle HD, De Cock KM. The Serostatus Approach to Fighting the HIV Epidemic: prevention strategies for infected individuals. Am J Public Health 2001; 91 (7): 1019-24.

16. UNAIDS. UNAIDS report on the global AIDS epidemic 2010. Disponible en: http://www.unaids.org/globalreport/global_report.htm

17. Wolitski RJ, Gómez CA, Parsons JT. Effects of a peer-led behavioral intervention to reduce HIV transmission and promote serostatus disclosure among HIV-seropositive gay and bisexual men. AIDS 2005; 19 Suppl 1: S99-109.

18. Kalichman S. HIV transmission risk behaviors of men and women living with HIV-AIDS. Clin Psychol Sci Pract 2000; 7 (1): 32-47.

19. Marks G, Crepaz N, Senterfitt JW, Janssen RS. Metaanalysis of high-risk sexual behavior in persons aware and unaware they are infected with HIV in the United States: implications for HIV prevention programs. J Acquir Immune Defic Syndr 2005; 39 (4): 446-53.

20. Reilly T, Woo G. Predictors of high-risk sexual behavior among people living with HIV/AIDS AIDS Behav 2001; 5 (3): 205-17.

21. Estrada BD, Trujillo S, Estrada AL. Supporting Healthy Alternatives through Patient Education: a theoretically driven HIV prevention intervention for persons living with HIV/AIDS. AIDS Behav 2007; 11 (5 Suppl): S95105.

22. Grimley DM, Bachmann LH, Jenckes MW, Erbelding EJ. Provider-delivered, theory-based, individualized prevention interventions for HIV positive adults receiving HIV comprehensive care. AIDS Behav 2007; 11 (5 Suppl): S39-47.

23. Teti M, Rubinstein S, Lloyd L, Aaron E, Merron-Brainerd J, Spencer S, et al. The Protect and Respect program: a sexual risk reduction intervention for women living with HIV/AIDS. AIDS Behav 2007; 11 (5 Suppl): S 106-16.

24. Knauz RO, Safren SA, O'Cleirigh C, Capistrant BD, Dris- 
kell JR, Aguilar D, et al. Developing an HIV-prevention intervention for HIV-infected men who have sex with men in HIV care: project enhance. AIDS Behav 2007; 11 (5 Suppl): S 117-26.

25. Lightfoot M, Rotheram-Borus MJ, Tevendale H. An HIV-preventive intervention for youth living with HIV. Behav Modif 2007; 31 (3): 345-63.

26. Healthy Living Project Team. Effects of a behavioral intervention to reduce risk of transmission among people living with HIV: the healthy living project randomized controlled study. J Acquir Immune Defic Syndr 2007; 44 (2): 213-21.

27. Fisher JD, Smith L. Secondary prevention of HIV infection: the current state of prevention for positives. Curr Opin HIV AIDS 2009; 4 (4): 279-87.

28. Cleary PD, Van Devanter N, Steilen M, Stuart A, Shipton-Levy R, McMullen W, et al. A randomized trial of an education and support program for HIV-infected individuals. AIDS 1995; 9 (11): 1271-8.

29. Kalichman SC, Rompa D, Cage M, DiFonzo K, Simpson $\mathrm{D}$, Austin J, et al. Effectiveness of an intervention to reduce HIV transmission risks in HIV-positive people. Am J Prev Med 2001; 21 (2): 84-92.

30. Patterson TL, Shaw WS, Semple SJ. Reducing the sexual risk behaviors of HIV+ individuals: outcome of a randomized controlled trial. Ann Behav Med 2003; 25 (2): 137-45.

31. Richardson JL, Milam J, McCutchan A, Stoyanoff S, Bolan R, Weiss J, et al. Effect of brief safer-sex counseling by medical providers to HIV-1 seropositive patients: a multi-clinic assessment. AIDS 2004; 18 (8): 1179-86.

32. O'Grady L. Meeting health information needs of people with HIV/AIDS: sources and means of collaboration. Health Info Libr J 2008; 25 (4): 261-9.

33. Mendias EP, Paar DP. Perceptions of health and self-care learning needs of outpatients with HIV/AIDS. J Community Health Nurs 2007; 24 (1): 49-64.

34. Nokes KM, Nwakeze PC. Assessing self-management information needs of persons living with HIV/AIDS. AIDS Patient Care STDS 2005; 19 (9): 607-13.

35. Henderson SJ, Bernstein LB, George DM, Doyle JP, Paranjape AS, Corbie-Smith G. Older women and HIV: how much do they know and where are they getting their information? J Am Geriatr Soc 2004; 52 (9): 154953.

36. Hogan TP, Palmer CL. Information preferences and practices among people living with HIV/AIDS: results from a nationwide survey. J Med Libr Assoc 2005; 93 (4): 431-9.

37. Fisher JD, Fisher WA, Cornman DH, Amico RK, Bryan A, Friedland GH. Clinician-delivered intervention during routine clinical care reduces unprotected sexual behavior among HIV-infected patients. J Acquir Immune Defic Syndr 2006; 41 (1): 44-52.

38. Crepaz N, Lyles CM, Wolitski RJ, Passin WF, Rama SM, Herbst JH, et al. Do prevention interventions reduce HIV risk behaviours among people living with HIV? A meta-analytic review of controlled trials. AIDS 2006; 20 (2): 143-57.

39. Cornman DH, Kiene SM, Christie S, Fisher WA, Shuper PA, Pillay S, et al. Clinic-based intervention reduces unprotected sexual behavior among HIV-infected patients in KwaZulu-Natal, South Africa: results of a pilot study. J Acquir Immune Defic Syndr 2008; 48 (5): 553-60. 